\title{
Resection of gliomas deemed inoperable by neurosurgeons based on preoperative imaging studies
}

\author{
Derek G. Southwell, MD, PhD, ${ }^{1}$ Harjus S. Birk, MD, ${ }^{2}$ Seunggu J. Han, MD, ${ }^{1}$ Jing Li, MD, ${ }^{1}$ \\ Jeffrey W. Sall, MD, PhD, ${ }^{3}$ and Mitchel S. Berger, MD1
}

Departments of ${ }^{1}$ Neurological Surgery and ${ }^{3}$ Anesthesia and Perioperative Care, and ${ }^{2}$ School of Medicine, University of California, San Francisco, California

OBJECTIVE Maximal safe resection is a primary objective in the management of gliomas. Despite this objective, surgeons and referring physicians may, on the basis of radiological studies alone, assume a glioma to be unresectable. Because imaging studies, including functional MRI, may not localize brain functions (such as language) with high fidelity, this simplistic approach may exclude some patients from what could be a safe resection. Intraoperative direct electrical stimulation (DES) allows for the accurate localization of functional areas, thereby enabling maximal resection of tumors, including those that may appear inoperable based solely on radiological studies. In this paper the authors describe the extent of resection (EOR) and functional outcomes following resections of tumors deemed inoperable by referring physicians and neurosurgeons.

METHODS The authors retrospectively examined the cases of 58 adult patients who underwent glioma resection within 6 months of undergoing a brain biopsy of the same lesion at an outside hospital. All patients exhibited unifocal supratentorial disease and preoperative Karnofsky Performance Scale scores $\geq 70$. The EOR and 6-month functional outcomes for this population were characterized.

RESULTS Intraoperative DES mapping was performed on $96.6 \%$ (56 of 58 ) of patients. Nearly half of the patients $(46.6 \%, 27$ of 58 ) underwent an awake surgical procedure with DES. Overall, the mean EOR was $87.6 \% \pm 13.6 \%$ (range $39.0 \%-100 \%$ ). Gross-total resection (resection of more than $99 \%$ of the preoperative tumor volume) was achieved in $29.3 \%$ (17 of 58 ) of patients. Subtotal resection (95\%-99\% resection) and partial resection (PR; < $95 \%$ resection) were achieved in $12.1 \%$ (7 of 58 ) and $58.6 \%$ (34 of 58 ) of patients, respectively. Of the cases that involved PR, the mean EOR was $79.4 \% \pm 12.2 \%$. Six months after surgery, no patient was found to have a new postoperative neurological deficit. The majority of patients $(89.7 \%, 52$ of 58$)$ were free of neurological deficits both pre- and postoperatively. The remainder of patients exhibited either residual but stable deficits $(5.2 \%, 3$ of 58$)$ or complete correction of preoperative deficits $(5.2 \%, 3$ of 58$)$.

CONCLUSIONS The use of DES enabled maximal safe resections of gliomas deemed inoperable by referring neurosurgeons. With rare exceptions, tumor resectability cannot be determined solely by radiological studies.

https://thejns.org/doi/abs/10.3171/2017.5.JNS17166

KEY WORDS glioma; resection; eloquent tumor; direct electrical stimulation; oncology

$\mathrm{D}$ ESPITE advances in operative and neoadjuvant therapeutics, diffuse gliomas remain a challenging disease entity to treat. Although we now understand diffuse gliomas as genetically and biologically heterogeneous, the management of these tumors begins with a common goal, i.e., to safely resect as much tumor as possible. For malignant gliomas, the current regimen, as established by the European Organization for Research and Treatment of Cancer/National Cancer Institute of Canada
Phase III trial, includes maximal safe surgical resection followed by external beam radiotherapy with concurrent and adjuvant temozolomide. ${ }^{43}$ Evidence across multiple large series clearly supports the benefit of greater extent of resection (EOR) for patients with newly diagnosed glioblastoma with respect to their overall survival and progression-free survival outcomes. ${ }^{24,25,38}$ Even at tumor recurrence, a more aggressive resection leads to improved long-term patient outcomes. ${ }^{2,4,18,26}$

ABBREVIATIONS ADP = afterdischarge potential; DES = direct electrical stimulation; $D T I=$ diffusion tensor imaging; EOR = extent of resection; GTR = gross-total resection; KPS = Karnofsky Performance Scale; MAC = minimum alveolar concentration; MSI = magnetic source imaging; PR = partial resection; STR = subtotal resection; TMS $=$ transcranial magnetic stimulation.

SUBMITTED January 17, 2017. ACCEPTED May 11, 2017.

INCLUDE WHEN CITING Published online November 10, 2017; DOI: 10.3171/2017.5.JNS17166. 
Similarly, for low-grade tumors, a greater EOR not only results in improved overall survival, but also delays malignant progression. ${ }^{20,21,30,41}$ In addition, seizure outcomes correlate with low-grade glioma EOR.${ }^{15}$ Finally, as for malignant gliomas, EOR correlates with overall survival in cases of low-grade glioma recurrence. ${ }^{32}$

Maximal tumor resection, however, must be balanced with preservation of the patient's neurological function. Postoperative neurological complications may impact patient quality of life, and they may also delay initiation of adjuvant therapy, thereby worsening survival as well.? Thus, tumors involving brain areas of presumed eloquent function have been considered "high risk" for resection in terms of potential for neurological morbidity. Multiple preoperative techniques have been developed to assist in identifying eloquent areas and their relationships to brain lesions, including functional MRI, diffusion tensor imaging (DTI), transcranial magnetic stimulation (TMS), magnetoencephalography, and magnetic source imaging (MSI). Intraoperative adjuncts, such as direct electrical stimulation (DES) mapping, intraoperative MRI, and 5-aminolevulinic acid, have also been used to maximize the safety of aggressive resection around eloquent areas. However, these techniques are not universally used and frequently only available at tertiary referral academic centers.

In the senior author's experience, patients are occasionally referred for consultation after undergoing biopsy or partial resection (PR) of a glioma at an outside hospital. In these cases the referring physician believed the tumor could not be safely resected due to its location on structural imaging studies or based on functional imaging studies that indicated function within or adjacent to the tumor. However, a craniotomy with functional mapping remains the definitive determinant of functional localization and, thus, the primary method for determining tumor resectability. If DES demonstrates no functional localization within the tumor, or within portions of the tumor, then resection is performed within the context of maximal safe resection. ${ }^{9,22}$ Thus, lesions viewed by some physicians as "inoperable" or "unresectable" based on imaging studies may very well be amenable to resection with the use of DES. In this paper we describe the senior author's experience with such tumors, with a focus on EOR and functional outcomes.

\section{Methods \\ Study Population}

We retrospectively examined the cases of 58 adult patients (aged 18 years or older) who presented to the University of California, San Francisco, Medical Center for evaluation of gliomas, after undergoing biopsies at outside hospitals. Each patient was drawn from a database maintained by the senior author (M.S.B.) and was included in this study if he or she met each of the following criteria: 1) at an outside hospital, the patient underwent diagnostic brain biopsy of a supratentorial glioma, which the referring providers had judged to be inoperable due to its location in or around eloquent regions; 2) upon referral to our institution, the patient's functional status was scored as $\geq$ 70 on the Karnofsky Performance Scale (KPS); 3) preop- erative volumetric MRI studies did not exhibit multifocal disease; 4) within 6 months from the date of the biopsy, the patient underwent primary resection of the glioma by the senior author; and 5) postoperative volumetric MRI studies were available. Patients were excluded if they had undergone any prior resection. All patients underwent tumor resection between August 2005 and July 2015. In some cases, patients were referred directly to our center by the neurosurgeon who performed the biopsy, while in others they were referred to a treating physician who then referred the patient to our center for reconsideration of resection. Some patients also presented to the senior author independently, seeking another opinion on resectability after being told that the glioma was "unresectable" by an outside neurosurgeon. The IRB at the University of California, San Francisco, approved the study.

\section{Preoperative and Postoperative Evaluations}

Patients were independently evaluated pre- and postoperatively (6 months after tumor resection) by at least two physicians (which included the attending neurosurgeon, resident neurosurgeon, or attending neurooncologist) using a standard neurological examination, which included assessments of sensorimotor and language functions. Sensory evaluation included testing of light touch, pain, and position sense, while motor evaluation included testing of facial movements and arm and leg strength (using a $0-5$ scale). Language evaluation included testing of object naming, repetition, reading, counting, and calculation. Any degree of sensory, motor, or language dysfunction was counted as a deficit. If there was a discrepancy between the examiners' assessments, the more severe deficit was used.

\section{Resection and Intraoperative Mapping}

All patients underwent preoperative volumetric MRI within 48 hours of surgery. These included volumetric T1-weighted, Gd-enhanced sequences; FLAIR sequences; and T2-weighted sequences. Tumors were identified preoperatively using MRI and, when available, magnetoencephalography and MR tractography (DTI or high angular resolution diffusion imaging) were used to identify surrounding brain structures.

Based on tumor location, the senior author elected to perform intraoperative DES to map eloquent sensory, motor, and/or language areas. DES was performed on 56 (96.6\%) of 58 patients. Awake intraoperative language mapping was performed on 27 (46.6\%) of 58 patients. Two patients did not undergo DES because the senior author believed that their tumors did not involve eloquent brain areas. The DES methods used here have been described previously. $22,36,37,42$

Craniotomies were made to expose either the tumor or brain tissue overlying the tumor and up to $2-3 \mathrm{~cm}$ of surrounding brain surface. For surgeries that involved language mapping, electrocorticography was used to measure stimulation-induced afterdischarge potentials (ADPs), thereby ensuring that mapping results were not affected by subclinical seizure activity. If DES caused seizures, the exposed brain surface was irrigated with chilled Ringer's solution to suppress ictal activity ${ }^{39}$ If seizures were refrac- 
tory to irrigation, intravenous propofol $(1 \mathrm{mg} / \mathrm{kg})$ was administered. Direct electrical stimulation was performed using 1-mm bipolar electrodes that were separated by a distance of $5 \mathrm{~mm}$. The electrodes delivered $60-\mathrm{Hz}$ biphasic square pulses (phase length $1.25 \mathrm{msec}$ ) in 4-second trains. The initial stimulation intensity was $1.5-2.5 \mathrm{~mA}$, which was then increased up to a maximum of $6 \mathrm{~mA}$ or, in language mapping cases, $1 \mathrm{~mA}$ less than that which evoked ADPs. ADPs were achieved with stimulation intensities that ranged between 2.5 and $6 \mathrm{~mA}$. The typical stimulation current was 3-4 mA. Stimulation sites were spaced over every square centimeter of exposed brain tissue surface. Stimulation intensities were recorded for each patient's procedure.

When more than 1 functional modality (i.e., sensory, motor, or language) was tested, mapping proceeded in the following sequence: sensory, motor, then language. A positive sensory site was identified if the patient reported focal paresthesias of the face, trunk, arm, or leg during application of the stimulus. A positive motor site was identified by involuntary passive movement of the face, arm, or leg or impaired motor function during active movement. If awake intraoperative language mapping was performed, the patient underwent preoperative language evaluation by a neuropsychologist. This evaluation included tests of object naming, reading, counting, calculation, and visuospatial identification. Intraoperative language testing was performed using only the items the individual patient had correctly identified during preoperative evaluation. Intraoperative language testing was performed cooperatively by the neuropsychologist and attending neurosurgeon. Positive language sites were defined by stimulation-induced anomia, alexia, or speech arrest, in the absence of motor or seizure activity, during 2 of 3 stimulation trials. ${ }^{37}$ Speech arrest was defined as disturbance of number counting (without involuntary mouth or pharyngeal muscle movement), anomia was defined as the inability to name sequential objects presented as line drawings, and alexia was defined as the inability to read words.

Stimulation sites were labeled with plastic markers, and the corresponding DES results at each site were transcribed onto a hand-drawn map and recorded in the operative report. The labeled mapping sites were recorded by digital photography prior to and following tumor resection.

Tumor resection was performed using frameless navigational guidance based on the preoperative MRI. Tumor resection was performed up to the margin of positive stimulation sites, as identified by DES. All nonfunctional tumor, as defined by DES, was resected.

\section{Neuroanesthesia}

For craniotomy with asleep mapping, standard monitors and an arterial line were used. Anesthesia induction was initiated with fentanyl in divided doses prior to and during induction $(1.5-4 \mu \mathrm{g} / \mathrm{kg})$, as well as lidocaine (40 $\mathrm{mg}$ ), propofol $(2-2.5 \mathrm{mg} / \mathrm{kg})$, rocuronium $(0.6-1 \mathrm{mg} / \mathrm{kg})$, or other relaxant. The muscle relaxant was allowed to wear off prior to motor mapping and was rarely reversed. Anesthesia was maintained using $70 \%$ nitrous oxide, 0.5 minimum alveolar concentration (MAC) or less of a volatile agent, and fentanyl infusion $(2 \mu \mathrm{g} / \mathrm{kg} / \mathrm{hr})$. Propofol was generally avoided but kept available for immediate intravenous administration in case of prolonged after-depolarizations with gross movement or seizure. Once motor mapping was completed, neuromuscular relaxation was initiated, along with propofol or an increased dose of a volatile agent. Labetalol was often required at the conclusion of surgery to prevent hypertension during emergence from anesthesia.

For craniotomy with asleep somatosensory mapping, nitrous oxide and halogenated volatile anesthetics were used minimally or avoided, as these agents can inhibit somatosensory evoked potentials. Anesthesia was maintained with 0.1-0.3 MAC of volatile anesthetic, propofol $(75-150 \mu \mathrm{g} / \mathrm{kg} / \mathrm{min})$, and fentanyl $(2 \mu \mathrm{g} / \mathrm{kg} / \mathrm{hr})$ as tolerated to maintain adequate signals. Neuromuscular relaxation was maintained with rocuronium or vecuronium. Obtaining a good baseline prior to starting the mapping is key.

For sedation during awake mapping procedures, propofol $(50-100 \mu \mathrm{g} / \mathrm{kg} / \mathrm{min})$ or dexmedetomidine (0.7-2.0 $\mu \mathrm{g} / \mathrm{kg} /$ hour), as well as a short-acting opiate such as remifentanil $(0.05-0.1 \mu \mathrm{g} / \mathrm{kg} / \mathrm{min})$, were used. Sedation was withheld following the craniotomy, prior to dural incision.

For awake mapping procedures, patients received midazolam $(0.5-2 \mathrm{mg})$ in the preoperative area, if necessary, to reduce anxiety. Once in the operating room sedation was started, and an arterial line, additional intravenous line, and Foley catheter were placed. A bolus of propofol or remifentanil was used to facilitate placement of the patient's head into a head holder. Adequate local anesthesia (lidocaine) was placed in the scalp. Intraoperative sedation was maintained by intravenous agents with short half-lives; propofol (up to $100 \mathrm{~g} / \mathrm{kg} / \mathrm{min}$ ) with remifentanil $(0.01-0.08 \mu \mathrm{g} / \mathrm{kg} / \mathrm{min})$ were generally adequate. Doses were titrated gradually to avoid respiratory depression. Dexmedetomidine $(0.3-1.5 \mu \mathrm{g} / \mathrm{kg} / \mathrm{hr}$ after $0.7 \mu \mathrm{g} / \mathrm{kg}$ bolus given over 10 minutes) was substituted for propofol after the mapping was completed, or throughout the surgeries in those patients for whom airway patency was a concern (for example, due to obesity).

\section{Tumor Volume and EOR}

Postoperative MRI was performed within the first 48 hours after surgery. Tumor volumes were analyzed preand postoperatively by measuring, in the axial plane, the area of the hyperintense mass on T2-weighted FLAIR sequences (for low-grade gliomas) or on T1-weighted contrast-enhanced MR images (for high-grade gliomas). Tumors were segmented manually across all slices, and the area of tumor in each slice was multiplied by the slice thickness to determine its slice volume. Tumor slice volumes were summed across all slices that contained tumor to determine total tumor volume.

The tumor EOR was determined by comparing tumor volumes between the pre- and postoperative MR images. The EOR was calculated using the following formula: [(postoperative tumor volume - preoperative tumor volume)/preoperative tumor volume] $\times 100$. For contrast-enhancing tumors, volumes were determined by measuring the contrast-enhancing volume on T1-weighted sequences. For nonenhancing tumors, the FLAIR or T2-weighted sequences were used for volume measurements. Gross-total 
resection (GTR) was considered EOR $>99 \%$, subtotal resection (STR) 95\%-99\%, and PR < 95\%.

\section{Statistical Analyses}

Data are presented as means \pm standard deviations. The Fisher's exact test (2-tailed) was used for assessment of categorical data, and the Student t-test (2-tailed) was used for continuous data. Statistical significance was set at $\mathrm{p}<$ 0.05 .

\section{Results \\ Demographic, Clinical, and Oncological Features of the Study Population}

Fifty-eight patients met the inclusion criteria for this study. Table 1 summarizes the patient population. Men comprised $70.7 \%$ of the study population (41 of 58 patients), while women comprised $29.3 \%$ (17 of 58). Nearly all patients $(94.8 \%, 55$ of 58$)$ were right-handed. The mean patient age at the time of resection was $43.1 \pm 13.2$ years (range 22-67 years). The mean interval between outside hospital biopsy and tumor resection was $63.2 \pm 33.4$ days (range 19-182 days). No patient underwent nonsurgical treatment (i.e., medical or radiation therapy) between biopsy and tumor resection. Seizures were the most common presenting complaint ( 49 of $58,84.5 \%$ ), followed by headache (5 of $58,8.6 \%$ ), altered mental status ( 3 of $58,5.2 \%$ ), gait disturbance ( 3 of 58,5.2\%), language disturbance ( 2 of $58,3.4 \%$ ), and focal motor weakness (1 of 58, 1.7\%).

Tumors involved the frontal lobe $(53.4 \%, 31$ of 58$)$, temporal lobe $(36.2 \%, 21$ of 58$)$, insula $(31.0 \%, 18$ of 58$)$, parietal lobe $(20.7 \%, 12$ of 58), cingulum (5.2\%, 3 of 58), and basal ganglia $(1.7 \%, 1$ of 58). Table 1 presents the presumed eloquence grading of the tumors, as described by Sawaya et al.:40 93.1\% of patients (54 of 58) exhibited Grade 2 or 3 tumors. Tumor histologies (at the time of resection) included glioblastoma $(29.3 \%, 17$ of 58 patients), oligodendroglioma (20.7\%, 12 of 58), anaplastic astrocytoma $(19.0 \%, 11$ of 58$)$, astrocytoma $(13.8 \%, 8$ of 58 ), oligoastrocytoma $(8.6 \%, 5$ of 58), anaplastic oligodendroglioma (5.2\%, 3 of 58), anaplastic oligoastrocytoma (1.7\%, 1 of 58), and pilocytic astrocytoma (1.7\%, 1 of 58). Pathology findings from resection were concordant with those from diagnostic biopsy in $81.0 \%$ of patients (47 of 58). In all 11 cases in which the findings differed, tumor grade was higher at the time of resection than at the time of biopsy.

\section{Preoperative and Intraoperative Anatomofunctional Studies}

To guide surgical planning, preoperative imaging studies were used to define tumor location relative to areas that may serve sensory, motor, or language functions (Table 2 ). Tractography of white matter fiber pathways was performed on $96.6 \%$ (56 of 58) of patients. To visualize tumor and predict language and motor areas, MR spectroscopy and MSI were used in $48.3 \%$ (28 of 58) and 36.2\% (21 of 58) of patients, respectively. However, neither MSI nor DTI were ever used to determine tumor resectability.

Intraoperative DES mapping was performed in $96.6 \%$ of patients (56 of 58; Table 2). Awake intraoperative mapping
TABLE 1. Demographic and oncological features of the study population

\begin{tabular}{|c|c|}
\hline Characteristic & Value $(\%)$ \\
\hline No. of patients & 58 \\
\hline Mean age at op in yrs $\pm S D$ & $43.1 \pm 13.2$ \\
\hline \multicolumn{2}{|l|}{ Sex } \\
\hline Male & $41(70.7)$ \\
\hline Female & $17(29.3)$ \\
\hline \multicolumn{2}{|l|}{ Handedness } \\
\hline Rt & $55(94.8)$ \\
\hline Lt & $3(5.2)$ \\
\hline \multicolumn{2}{|l|}{ Chief presenting complaint } \\
\hline Seizures & $49(84.5)$ \\
\hline Headache & $5(8.6)$ \\
\hline Altered mental status & $3(5.2)$ \\
\hline Gait disturbance & $3(5.2)$ \\
\hline Language disturbance & $2(3.4)$ \\
\hline Hand weakness & $1(1.7)$ \\
\hline \multicolumn{2}{|l|}{ Sidedness of tumor } \\
\hline $\mathrm{Lt}$ & $33(56.9)$ \\
\hline Rt & $24(41.4)$ \\
\hline Bilat & $1(1.7)$ \\
\hline \multicolumn{2}{|l|}{ Brain areas involved by tumor } \\
\hline Frontal lobe & $31(53.4)$ \\
\hline Temporal lobe & $21(36.2)$ \\
\hline Insula & $18(31.0)$ \\
\hline Parietal & $12(20.7)$ \\
\hline Cingulum & $3(5.2)$ \\
\hline Basal ganglia & $1(1.7)$ \\
\hline Mean interval btwn biopsy \& resection in days \pm SD & $63.2 \pm 33.4$ \\
\hline \multicolumn{2}{|l|}{ Presumed eloquence grade } \\
\hline I (tumor in presumed noneloquent area) & $4(6.9)$ \\
\hline II (tumor near presumed eloquent area) & $34(58.6)$ \\
\hline III (tumor in presumed eloquent area) & $20(34.5)$ \\
\hline \multicolumn{2}{|l|}{ Tumor pathology } \\
\hline Glioblastoma & $17(29.3)$ \\
\hline Oligodendroglioma & $12(20.7)$ \\
\hline Anaplastic astrocytoma & $11(19.0)$ \\
\hline Astrocytoma & $8(13.8)$ \\
\hline Oligoastrocytoma & $5(8.6)$ \\
\hline Anaplastic oligodendroglioma & $3(5.2)$ \\
\hline Anaplastic oligoastrocytoma & $1(1.7)$ \\
\hline Pilocytic astrocytoma & $1(1.7)$ \\
\hline
\end{tabular}

All data given as number of patients (\%) unless otherwise indicated.

was performed in $46.6 \%$ of patients (27 of 58). Intraoperative mapping was performed under general anesthesia in $50.0 \%$ of patients (29 of 58). As previously mentioned, 2 patients did not undergo DES mapping because the senior author felt that tumor resection would not occur within, or adjacent to, functional areas. Sensorimotor mapping of cortical tissues was performed in $60.3 \%$ of patients ( 35 of 58). Positive cortical sensorimotor sites were identified in 
TABLE 2. Preoperative and intraoperative functional studies

\begin{tabular}{cc}
\hline \multicolumn{1}{c}{ Characteristic } & No. of Patients $(\%)$ \\
\hline Preop functional study performed & \\
\hline Tractography & $56(96.6)$ \\
\hline Functional MR spectroscopy & $28(48.3)$ \\
\hline MSI & $21(36.2)$ \\
\hline Intraop DES & $56(96.6)$ \\
\hline Performed & $2(3.4)$ \\
\hline Not performed & \\
\hline Awake surgical procedure & $31(53.4)$ \\
\hline Not performed & $27(46.6)$ \\
\hline Performed & $35(60.3)$ \\
\hline Cortical sensorimotor mapping & $31(88.6)$ \\
\hline Negative result* & $4(11.4)$ \\
\hline Positive result $\dagger$ & $35(60.3)$ \\
\hline Subcortical sensorimotor mapping & $34(97.1)$ \\
\hline Negative result & $1(2.9)$ \\
\hline Positive result & $26(44.8)$ \\
\hline Cortical language mapping & $20(76.9)$ \\
\hline Negative result & $6(23.1)$ \\
\hline Positive result & $19(32.8)$ \\
\hline Subcortical language mapping & $19(100)$ \\
\hline Negative result & $0(0)$ \\
\hline Positive result &
\end{tabular}

* Negative result $=$ no eloquent tissue identified.

$\dagger$ Positive result $=$ eloquent tissue identified.

$11.4 \%$ of these patients (4 of 35). Sensorimotor mapping of subcortical tissues was also performed in $60.3 \%$ of patients (35 of 58). Positive subcortical sensorimotor sites were identified in $2.9 \%$ of these patients ( 1 of 35). Language mapping of cortical tissues was performed in $44.8 \%$ of patients (26 of 58). Positive cortical language sites were identified in $23.1 \%$ of patients (6 of 26). Language mapping of subcortical tissues was performed in $32.8 \%$ of patients (19 of 58). Positive subcortical language sites were identified in $0.0 \%$ of patients ( 0 of 19). Altogether, positive sites were identified in $19.6 \%$ of patients who underwent DES mapping (11 of 56). Tumor resection was aimed at removing all tumor volume, regardless of whether it was enhancing or nonenhancing. Resection was limited by the presence of positive sites (i.e., eloquent tissue) in $60 \%$ (6 of $10)$ of the cases in which they were identified.

\section{Extent of Resection and Postoperative Treatments}

The mean preoperative tumor volume was $48.6 \pm 47.1$ $\mathrm{cm}^{3}$ (range $0.2-244.3 \mathrm{~cm}^{3}$ ), and the mean postoperative volume was $7.9 \pm 10.4 \mathrm{~cm}^{3}$ (range $0.0-50.1 \mathrm{~cm}^{3}$; Table 3). Overall, the mean EOR was $87.6 \% \pm 13.6 \%$ (range $39.0 \%-$ $100 \%)$. For surgeries that involved motor mapping with the patient asleep $(\mathrm{n}=29)$, the mean EOR was $85.8 \% \pm$ $15.2 \%$ (range $39.0 \%-100 \%$ ). For surgeries that involved language mapping $(\mathrm{n}=27)$, the mean EOR was $88.8 \% \pm$ $11.9 \%$ (range $62.8 \%-100 \%$ ). Overall, GTR (resection of > $99 \%$ of the preoperative tumor volume) occurred in $29.3 \%$
TABLE 3. Functional outcomes, extent of resection, and postsurgical treatments

\begin{tabular}{|c|c|}
\hline Characteristic & Value \\
\hline \multicolumn{2}{|l|}{ Preop neurological deficit, $\mathrm{n}(\%)$} \\
\hline Absent & $52(89.7)$ \\
\hline Present & $6(10.3)$ \\
\hline Motor & $3(5.2)$ \\
\hline Sensory & $0(0)$ \\
\hline Language & $4(6.9)$ \\
\hline \multicolumn{2}{|l|}{ Postop neurological deficit, $n(\%)$} \\
\hline Absent & $55(94.8)$ \\
\hline Present & $3(5.2)$ \\
\hline Motor & $2(3.4)$ \\
\hline Sensory & $0(0)$ \\
\hline Language & $1(1.7)$ \\
\hline \multicolumn{2}{|c|}{$\begin{array}{l}\text { Comparison of post- \& preop neurological } \\
\quad \text { examinations, } n(\%)\end{array}$} \\
\hline Stable, w/o deficit & $52(87.9)$ \\
\hline Stable, w/ residual deficit & $3(5.2)$ \\
\hline Improved, w/o deficit & $3(5.2)$ \\
\hline Worsened & $0(0)$ \\
\hline \multicolumn{2}{|c|}{ Mean tumor volume $\pm \mathrm{SD}$ in $\mathrm{cm}^{3}$ (range) } \\
\hline Preop & $48.6 \pm 47.1(0.2-244.3)$ \\
\hline Postop & $7.9 \pm 10.4(0.0-50.1)$ \\
\hline \multicolumn{2}{|l|}{ EOR, $n(\%)$} \\
\hline GTR (>99\%) & $17(29.3)$ \\
\hline STR $(95 \%-99 \%)$ & $7(12.1)$ \\
\hline PR (<95\%) & $34(58.6)$ \\
\hline \multicolumn{2}{|l|}{ Postop treatments, $\mathrm{n}(\%)$} \\
\hline Additional resection & $16(27.6)$ \\
\hline Radiation + temozolamide & $23(39.7)$ \\
\hline Temozolamide & $11(19.0)$ \\
\hline None & $4(6.9)$ \\
\hline Bevacizumab & $2(3.4)$ \\
\hline Temozolamide + bevacizumab & $1(1.7)$ \\
\hline Temozolamide + lomustine & $1(1.7)$ \\
\hline
\end{tabular}

of patients (17 of 58). STR (95\%-99\% resection) and PR (< $95 \%$ resection) occurred in $12.1 \%$ (7 of 58) and $58.6 \%$ of patients (34 of 58), respectively. Of the cases that involved $\mathrm{PR}$, the mean EOR was $79.4 \% \pm 12.2 \%$. Representative cases of low-grade and high-grade glioma resections are depicted in Figs. 1-3.

Nonsurgical postoperative treatments included combined radiotherapy and temozolomide $(39.7 \%, 23$ of 58 patients), temozolomide $(19.0 \%, 11$ of 58$)$, bevacizumab (3.4\%, 2 of 58), and dual-agent chemotherapy (temozolomide and bevacizumab; $1.7 \%, 1$ of 58; and temozolomide and lomustine $(1.7 \%, 1$ of 58). Within 6 months of their initial surgeries, 2 patients $(3.4 \%, 2$ of 58$)$ underwent reoperations at our institution for tumor resection; 1 patient with glioblastoma underwent reoperation 3 months after his initial resection, and another patient with anaplastic astrocytoma underwent reoperation 5 months after his 

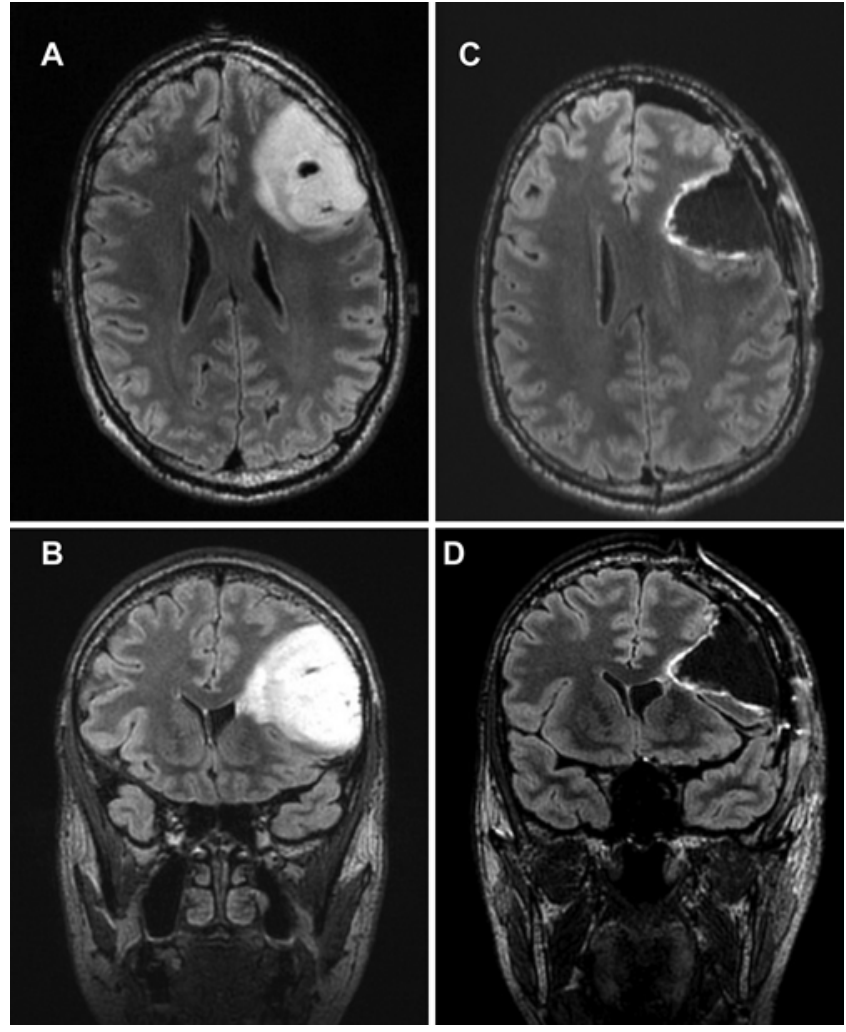

FIG. 1. Representative case involving a 31-year-old right-handed man who presented with complaints of seizures. The biopsy performed at an outside hospital indicated Grade II astrocytoma. Preoperative FLAIR MRI sequences (axial slice, A; coronal slice, B) depicted a nonenhancing lesion involving the left frontal lobe. Resection was performed using awake cortical and subcortical mapping of language function, as well as awake cortical sensorimotor mapping; cortical language mapping identified 1 positive site. A GTR (EOR 99\%) was achieved, as demonstrated by the postoperative MRI (axial slice, C; coronal slice, D). The patient exhibited intact neurological function 6 months after surgery.

initial resection. Fourteen patients $(24.1 \%, 14$ of 58$)$ underwent additional resection after the 6-month follow-up period; for this group, the mean interval between initial surgery and reoperation was $49.2 \pm 29.4$ months (the range of follow-up for this group was 29-125 months).

\section{Neurological Outcomes}

Preoperatively, $10.3 \%$ of patients (6 of 58) exhibited neurological deficits (Table 3): motor deficits were noted in $5.2 \%$ of patients ( 3 of 58), while language deficits were noted in $6.9 \%$ (4 of 58). No patient exhibited a preoperative sensory deficit. Six months after tumor resection, $5.2 \%$ of patients ( 3 of 58) exhibited neurological deficits. Motor deficits were present in $3.4 \%$ of patients ( 2 of 58 ), and language deficits were present in $1.7 \%$ of patients (1 of 58). No patient exhibited a postoperative sensory deficit.

Six months after resection, $93.1 \%$ of patients (54 of 58) exhibited stable neurological examination findings, as compared with their preoperative examinations (Table 3). The majority of patients $(89.7 \%, 52$ of 58 ) were free of neurological deficits both pre- and postoperatively. Smaller numbers of patients exhibited residual, but stable, deficits (5.2\%, 3 of 58). The remainder of patients $(5.2 \%, 3$ of 58)
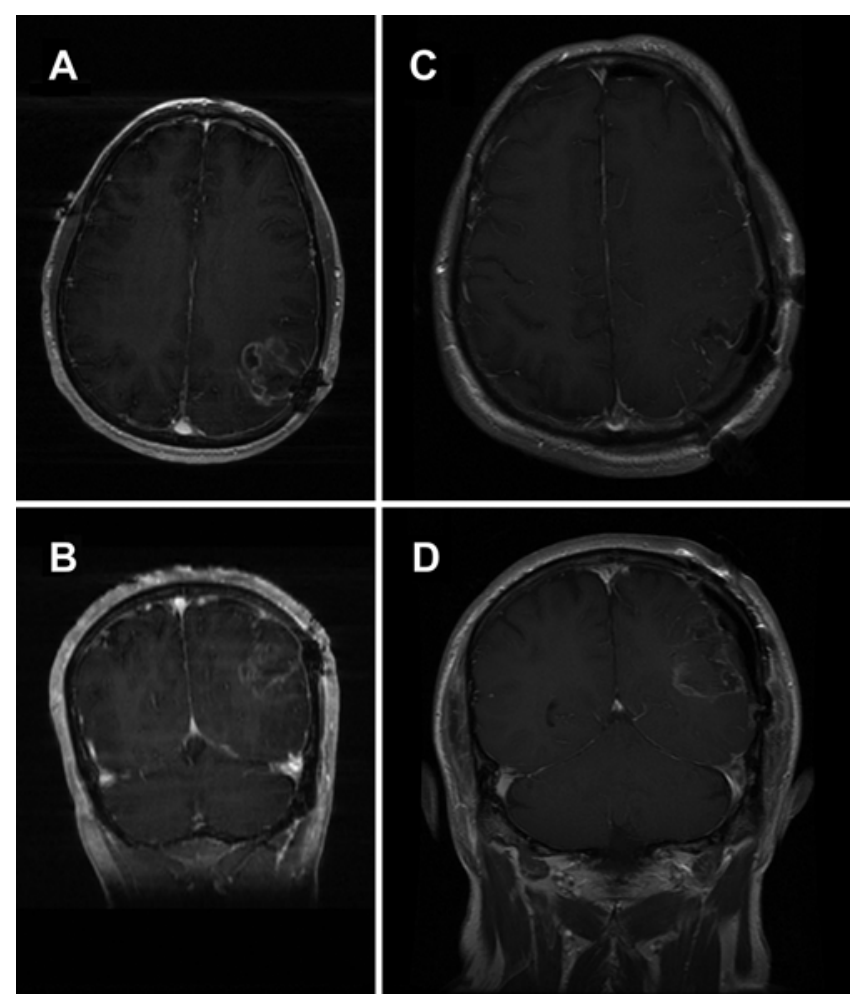

FIG. 2. Representative case involving a 52-year-old right-handed man who presented with complaints of seizures and language disturbances (mild expressive aphasia). The biopsy performed at an outside hospital indicated glioblastoma. Preoperative T1-weighted Gd MRI sequences (axial slice, A; coronal slice, B) depicted an enhancing lesion involving the left parietal lobe. Resection of the lesion was performed using awake cortical and subcortical mapping of language function; no positive language sites were identified. A GTR (EOR 99\%) was performed, as demonstrated by the postoperative MRI (axial slice, C; coronal slice, D). The patient exhibited improved and intact neurological function 6 months after surgery.

experienced resolution of their preoperative deficits. No patient was found to have a new postoperative neurological deficit. Six of 58 patients (10.3\%) exhibited transient postoperative deficits that resolved within 6 months of surgery. Three patients exhibited transient speech deficits (speech hesitation in 1, naming difficulty in 2), and 3 patients exhibited mild weakness.

\section{Discussion}

Resection is a central component of low- and highgrade glioma treatment. Numerous studies suggest that, regardless of tumor grade, patient outcomes correlate with the tumor EOR. ${ }^{17,19}$ Due to their infiltrative nature, gliomas often involve or abut gray- and white-matter tissues that serve sensorimotor or language functions. In these cases, tumor "resectability" can be limited by the risk of iatrogenic injury during resection. Unfortunately, surgeons commonly ascertain this risk by using MRI to assess the lesion's proximity to presumed eloquent areas. This practice, however, likely precludes what, in many cases, could in fact be a safe resection..$^{13,27}$

In the current study we evaluated EOR and neurological outcomes in patients who underwent glioma resection 

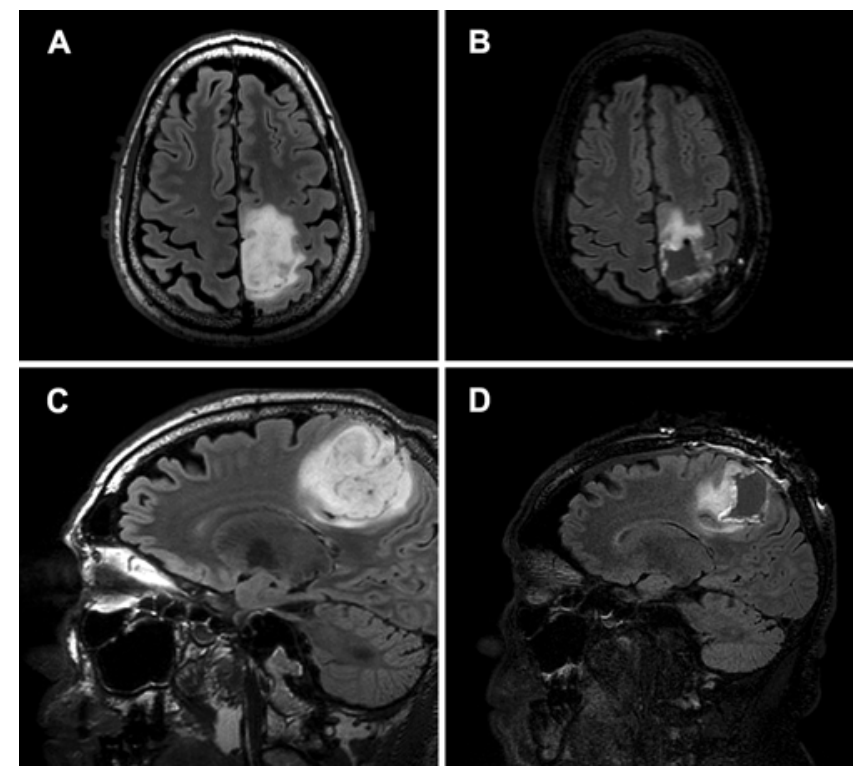

FIG. 3. Representative case involving a 61-year-old right-handed man who presented with complaints of seizures and right foot weakness. The outside hospital biopsy indicated Grade II astrocytoma. Preoperative FLAIR MRI sequences (axial slice, A; sagittal slice, C) depicted a nonenhancing lesion involving the left frontal and parietal lobes. Resection was performed using cortical and subcortical mapping of motor function, both of which demonstrated positive sites in and around the lesion. A PR (EOR 75\%) was achieved, as demonstrated by the postoperative MRI (axial slice, B; sagittal slice, D). The patient exhibited stable neurological function 6 months after surgery (i.e., persistent, but not worsened, right foot weakness).

at our hospital following biopsy of the same lesion by an outside neurosurgeon. These patients were not offered resections at the outside facilities because their tumors were assumed to be inoperable on the basis of imaging studies. In all cases, the patients' radiological studies suggested the glioma might involve or be contiguous with eloquent brain areas. Patients were referred either by neurosurgeons who do not perform intraoperative brain mapping or by those who perform mapping yet believed the patient's tumor could not be safely resected. In addition, some patients were referred by neuroradiologists or radiation oncologists who questioned whether the tumors were inoperable. Finally, some patients presented to the senior author unwilling to accept the outside neurosurgeon's opinion regarding resectability. The senior author chose to attempt tumor resection in each patient presented here, and he performed mapping in $96.6 \%$ of cases, with the goal of removing as much of the tumor as safely as possible before initiating nonsurgical treatments. Awake mapping was performed during $46.6 \%$ of the resections (27 of 58).

Despite the lesions being deemed inoperable by referring physicians, surgical treatment was undertaken, and it yielded a mean EOR of $87.6 \% \pm 13.6 \%$. An EOR $>95 \%$ was achieved for $41.4 \%$ of patients (24 of 58), and GTR (> $99 \%$ ) was achieved for $29.3 \%$ (17 of 58 patients). The EOR was $<75 \%$ for only $12.1 \%$ of patients (7 of 58). In addition to significantly reducing tumor volume, resection was also remarkably safe: 6 months after surgery, no patient exhibited a new postoperative neurological deficit. Preoperative neurological deficits were improved by resection in $5.2 \%$ of patients ( 3 of 58). The remainder of patients exhibited either stable, intact postoperative neurological function (52 of $58,89.7 \%$ ) or no change in their preoperative neurological deficits (3 of 58, 5.2\%). EOR significantly differed between patients who did not experience improvement of their neurological deficits, as compared with those who experienced resolution of their neurological deficits $(67.2 \% \pm$ $6.8 \%$ vs $91.3 \% \pm 10.0 \%$, respectively; $\mathrm{p}=0.02$ ).

The outcomes observed in this study align with limited work from others showing high EORs and mild postoperative morbidity rates for patients who underwent resections of "intractable" gliomas. ${ }^{16,23}$ Additionally, our findings are consistent with the senior author's previous work demonstrating minimal neurological morbidity following glioma resection within and around perisylvian regions and rolandic cortex. ${ }^{22,37}$ Altogether, the current study demonstrates that, for patients with good preoperative functional status (KPS score $\geq 70$ ) and unifocal, supratentorial disease, the use of intraoperative brain mapping allows for the safe resection of so-called "inoperable" gliomas.

There is solid evidence that intraoperative stimulation mapping reduces permanent neurological deficits without compromising extent of glioma resection..$^{9,14}$ This is true despite the fact that mapping is typically selected for procedures involving high-risk brain areas. In the current study, intraoperative stimulation mapping was performed on nearly every patient $(96.6 \%, 56$ of 58), and it explains why a high EOR could be achieved without neurological morbidity. Overall our results support the assertion that intraoperative stimulation mapping should be considered a standard of care for glioma resection. ${ }^{9}$ Furthermore, they support other studies demonstrating the importance of provider volume and care centralization for enhancing neurosurgical outcomes in patients with brain tumors. ${ }^{1,8}$

Our findings overwhelmingly demonstrate that structural and functional imaging cannot be used to determine glioma resectability, at least for lesions involving cortical or adjacent subcortical tissues. Despite being deemed inoperable by other physicians on the basis of MR images, all lesions described here were resected without causing new morbidity. Despite presumed tumor involvement of eloquent areas preoperatively, stimulation mapping rarely identified functional tissue involved within, or adjacent to, the glioma. When performed, cortical and subcortical sensorimotor mapping identified positive sites in only $11.4 \%$ and $2.9 \%$ of cases, respectively. Likewise, cortical and subcortical language mapping identified positive sites in $23.1 \%$ and $0 \%$ of patients, respectively. Thus, while other neurosurgeons believed these gliomas likely involved or apposed critical brain areas, intraoperative stimulation mapping during the subsequent resections rarely showed this to be correct. While other noninvasive, presurgical modalities such as functional MRI, TMS, magnetoencephalography, and diffusion tractography may become increasingly useful adjuncts for localizing eloquent areas and preoperatively assessing surgical risk, intraoperative DES is currently the most accurate, robust, and available method for identifying functional brain tissue. $3,11,28,29,33,44-46$ While tractography was helpful here for defining white matter pathways and guiding the use of subcortical DES, resection limits were ultimately defined by DES alone. 
The location of a glioma, as depicted by radiological studies, including functional imaging, may be used to determine the need for intraoperative stimulation mapping, but it should not necessarily be used to deem a glioma inoperable; this is particularly true for gliomas presumed to involve language areas.

Why were these presumably "inoperable," i.e., eloquent area gliomas largely amenable to safe resection? Likewise, why were relatively few functional sites identified by stimulation mapping during resection of these tumors, even though preoperative imaging suggested the tumors infiltrated or abutted functional tissues? One plausible explanation is that preoperative assessments of tumor resectability were based on classic but simplistic models of brain functional organization, which assume that patients exhibit a conserved and immutable localization of sensorimotor and language functions..$^{31,48}$ This "single brain model" has led to the notion of a generic and conserved organization of eloquent brain areas, wherein resection is expected to cause neurological morbidity. Such traditional and simplistic models, however, do not take into account growing evidence for both individual variability in functional localization, as well as the diffuse and dynamic organization of neural networks, particularly those serving language functions..$^{5,6,13,47}$ Recent evidence from neurosurgical patient studies suggest that brain plasticity allows for the reorganization of function, particularly across cortical tissues..$^{10,12,34,35,42}$ Patients in our study population may have undergone such reorganizations, thereby limiting the effectiveness of neuroimaging for predicting the risk of resection of their tumors. In support of this possibility, very few of our patients $(10.3 \%, 6$ of 58) exhibited preoperative neurological deficits, even though their gliomas involved or apposed presumably eloquent structures.

\section{Conclusions}

Our findings support a strategy for glioma treatment by which all patients with good functional status (KPS score $\geq 70$ ) and unifocal, supratentorial disease are offered resection, regardless of whether imaging shows the tumor abutting or involving presumed eloquent areas. In these cases, intraoperative stimulation mapping must be performed to localize function and achieve maximal safe resection. Preoperative anatomical or functional imaging should not be used to determine the operability of unifocal, supratentorial gliomas. The tendency to a priori rule out resection based on neuroimaging compromises patient outcomes by leading to the underutilization of resection. ${ }^{23}$

\section{Acknowledgments}

Dr. Birk was supported by a medical research fellowship from the Howard Hughes Medical Institute.

\section{References}

1. Barker FG II, Curry WT Jr, Carter BS: Surgery for primary supratentorial brain tumors in the United States, 1988 to 2000: the effect of provider caseload and centralization of care. Neuro Oncol 7:49-63, 2005

2. Bloch O, Han SJ, Cha S, Sun MZ, Aghi MK, McDermott
MW, et al: Impact of extent of resection for recurrent glioblastoma on overall survival: clinical article. J Neurosurg 117:1032-1038, 2012

3. Bush NAO, Chang SM, Berger MS: Current and future strategies for treatment of glioma. Neurosurg Rev 40:1-14, 2017, 2016

4. Chaichana KL, Zadnik P, Weingart JD, Olivi A, Gallia GL, Blakeley J, et al: Multiple resections for patients with glioblastoma: prolonging survival. J Neurosurg 118:812-820, 2013

5. Chang EF, Breshears JD, Raygor KP, Lau D, Molinaro AM, Berger MS: Stereotactic probability and variability of speech arrest and anomia sites during stimulation mapping of the language dominant hemisphere. J Neurosurg 126:114-121, 2016

6. Chang EF, Raygor KP, Berger MS: Contemporary model of language organization: an overview for neurosurgeons. J Neurosurg 122:250-261, 2015

7. Chang SM, Parney IF, McDermott M, Barker FG II, Schmidt $\mathrm{MH}$, Huang W, et al: Perioperative complications and neurological outcomes of first and second craniotomies among patients enrolled in the Glioma Outcome Project. J Neurosurg 98:1175-1181, 2003

8. Cowan JA Jr, Dimick JB, Leveque JC, Thompson BG, Upchurch GR Jr, Hoff JT: The impact of provider volume on mortality after intracranial tumor resection. Neurosurgery 52:48-54, 2003

9. De Witt Hamer PC, Robles SG, Zwinderman AH, Duffau H, Berger MS: Impact of intraoperative stimulation brain mapping on glioma surgery outcome: a meta-analysis. J Clin Oncol 30:2559-2565, 2012

10. Duffau H: Diffuse low-grade gliomas and neuroplasticity. Diagn Interv Imaging 95:945-955, 2014

11. Duffau H: Diffusion tensor imaging is a research and educational tool, but not yet a clinical tool. World Neurosurg 82:e43-e45, 2014

12. Duffau H: The huge plastic potential of adult brain and the role of connectomics: new insights provided by serial mappings in glioma surgery. Cortex 58:325-337, 2014

13. Duffau H: A two-level model of interindividual anatomofunctional variability of the brain and its implications for neurosurgery. Cortex 86:303-313, 2017

14. Duffau H, Lopes M, Arthuis F, Bitar A, Sichez JP, Van Effenterre R, et al: Contribution of intraoperative electrical stimulations in surgery of low grade gliomas: a comparative study between two series without (1985-96) and with (1996-2003) functional mapping in the same institution. J Neurol Neurosurg Psychiatry 76:845-851, 2005

15. Englot DJ, Berger MS, Barbaro NM, Chang EF: Predictors of seizure freedom after resection of supratentorial low-grade gliomas. A review. J Neurosurg 115:240-244, 2011

16. Ferroli P, Schiariti M, Finocchiaro G, Salmaggi A, Castiglione M, Acerbi F, et al: Operability of glioblastomas: "sins of action" versus "sins of non-action". Neurol Sci 34:21072116, 2013

17. Hervey-Jumper SL, Berger MS: Maximizing safe resection of low- and high-grade glioma. J Neurooncol 130:269-282, 2016

18. Hervey-Jumper SL, Berger MS: Reoperation for recurrent high-grade glioma: a current perspective of the literature. Neurosurgery 75:491-499, 2014

19. Hervey-Jumper SL, Berger MS: Role of surgical resection in low- and high-grade gliomas. Curr Treat Options Neurol 16:284, 2014

20. Ius T, Isola M, Budai R, Pauletto G, Tomasino B, Fadiga L, et al: Low-grade glioma surgery in eloquent areas: volumetric analysis of extent of resection and its impact on overall survival. A single-institution experience in 190 patients: clinical article. J Neurosurg 117:1039-1052, 2012 
21. Jakola AS, Unsgård G, Myrmel KS, Kloster R, Torp SH, Lindal S, et al: Low grade gliomas in eloquent locations - implications for surgical strategy, survival and long term quality of life. PLoS One 7:e51450, 2012

22. Keles GE, Lundin DA, Lamborn KR, Chang EF, Ojemann G, Berger MS: Intraoperative subcortical stimulation mapping for hemispherical perirolandic gliomas located within or adjacent to the descending motor pathways: evaluation of morbidity and assessment of functional outcome in 294 patients. J Neurosurg 100:369-375, 2004

23. Krieg SM, Schnurbus L, Shiban E, Droese D, Obermueller $\mathrm{T}$, Buchmann N, et al: Surgery of highly eloquent gliomas primarily assessed as non-resectable: risks and benefits in a cohort study. BMC Cancer 13:51, 2013

24. Lacroix M, Abi-Said D, Fourney DR, Gokaslan ZL, Shi W, DeMonte F, et al: A multivariate analysis of 416 patients with glioblastoma multiforme: prognosis, extent of resection, and survival. J Neurosurg 95:190-198, 2001

25. Marko NF, Weil RJ, Schroeder JL, Lang FF, Suki D, Sawaya RE: Extent of resection of glioblastoma revisited: personalized survival modeling facilitates more accurate survival prediction and supports a maximum-safe-resection approach to surgery. J Clin Oncol 32:774-782, 2014

26. Oppenlander ME, Wolf AB, Snyder LA, Bina R, Wilson JR, Coons SW, et al: An extent of resection threshold for recurrent glioblastoma and its risk for neurological morbidity. J Neurosurg 120:846-853, 2014

27. Orringer D, Lau D, Khatri S, Zamora-Berridi GJ, Zhang K, $\mathrm{Wu} \mathrm{C}$, et al: Extent of resection in patients with glioblastoma: limiting factors, perception of resectability, and effect on survival. J Neurosurg 117:851-859, 2012

28. Ostrý S, Belšan T, Otáhal J, Beneš V, Netuka D: Is intraoperative diffusion tensor imaging at 3.0T comparable to subcortical corticospinal tract mapping? Neurosurgery 73:797-807, 2013

29. Ottenhausen M, Krieg SM, Meyer B, Ringel F: Functional preoperative and intraoperative mapping and monitoring: increasing safety and efficacy in glioma surgery. Neurosurg Focus 38(1):E3, 2015

30. Pallud J, Taillandier L, Capelle L, Fontaine D, Peyre M, Ducray F, et al: Quantitative morphological magnetic resonance imaging follow-up of low-grade glioma: a plea for systematic measurement of growth rates. Neurosurgery 71:729-740, 2012

31. Penfield W, Boldrey E: Somatic motor and sensory representation in the cerebral cortex of man as studied by electrical stimulation. Brain 60:389-443, 1937

32. Ramakrishna R, Hebb A, Barber J, Rostomily R, Silbergeld D: Outcomes in reoperated low-grade gliomas. Neurosurgery 77:175-184, 2015

33. Roberts TP, Ferrari P, Perry D, Rowley HA, Berger MS: Presurgical mapping with magnetic source imaging: comparisons with intraoperative findings. Brain Tumor Pathol 17:57-64, 2000

34. Robles SG, Gatignol P, Lehéricy S, Duffau H: Long-term brain plasticity allowing a multistage surgical approach to World Health Organization Grade II gliomas in eloquent areas. J Neurosurg 109:615-624, 2008

35. Saito T, Muragaki Y, Miura I, Tamura M, Maruyama T, Nitta $\mathrm{M}$, et al: Functional plasticity of language confirmed with intraoperative electrical stimulations and updated neuronavigation: case report of low-grade glioma of the left inferior frontal gyrus. Neurol Med Chir (Tokyo) 54:587-592, 2014

36. Sanai N, Berger MS: Intraoperative stimulation techniques for functional pathway preservation and glioma resection. Neurosurg Focus 28(2):E1, 2010

37. Sanai N, Mirzadeh Z, Berger MS: Functional outcome after language mapping for glioma resection. N Engl J Med 358:18-27, 2008
38. Sanai N, Polley MY, McDermott MW, Parsa AT, Berger MS: An extent of resection threshold for newly diagnosed glioblastomas. J Neurosurg 115:3-8, 2011

39. Sartorius CJ, Berger MS: Rapid termination of intraoperative stimulation-evoked seizures with application of cold Ringer's lactate to the cortex. Technical note. J Neurosurg 88:349351,1998

40. Sawaya R, Hammoud M, Schoppa D, Hess KR, Wu SZ, Shi WM, et al: Neurosurgical outcomes in a modern series of 400 craniotomies for treatment of parenchymal tumors. Neurosurgery 42:1044-1056, 1998

41. Smith JS, Chang EF, Lamborn KR, Chang SM, Prados MD, Cha S, et al: Role of extent of resection in the long-term outcome of low-grade hemispheric gliomas. J Clin Oncol 26:1338-1345, 2008

42. Southwell DG, Hervey-Jumper SL, Perry DW, Berger MS: Intraoperative mapping during repeat awake craniotomy reveals the functional plasticity of adult cortex. J Neurosurg 124:1460-1469, 2016

43. Stupp R, Mason WP, van den Bent MJ, Weller M, Fisher $\mathrm{B}$, Taphoorn MJ, et al: Radiotherapy plus concomitant and adjuvant temozolomide for glioblastoma. N Engl J Med 352:987-996, 2005

44. Tarapore PE, Findlay AM, Honma SM, Mizuiri D, Houde JF, Berger MS, et al: Language mapping with navigated repetitive TMS: proof of technique and validation. Neuroimage 82:260-272, 2013

45. Tarapore PE, Martino J, Guggisberg AG, Owen J, Honma SM, Findlay A, et al: Magnetoencephalographic imaging of resting-state functional connectivity predicts postsurgical neurological outcome in brain gliomas. Neurosurgery 71:1012-1022, 2012

46. Tarapore PE, Tate MC, Findlay AM, Honma SM, Mizuiri D, Berger MS, et al: Preoperative multimodal motor mapping: a comparison of magnetoencephalography imaging, navigated transcranial magnetic stimulation, and direct cortical stimulation. J Neurosurg 117:354-362, 2012

47. Tate MC, Herbet G, Moritz-Gasser S, Tate JE, Duffau H: Probabilistic map of critical functional regions of the human cerebral cortex: Broca's area revisited. Brain 137:27732782, 2014

48. Wernicke C: The aphasic-symptom complex: a psychological study on an anatomical basis. Arch Neurol 22:280-282, 1970

\section{Disclosures}

The authors report no conflict of interest concerning the materials or methods used in this study or the findings specified in this paper.

\section{Author Contributions}

Conception and design: Southwell, Berger. Acquisition of data: Southwell, Birk, Han, Li. Analysis and interpretation of data: Southwell, Han, Berger. Drafting the article: Southwell, Birk, Han, Sall, Berger. Critically revising the article: Southwell, Han, Berger. Reviewed submitted version of manuscript: Southwell, Berger. Approved the final version of the manuscript on behalf of all authors: Southwell. Statistical analysis: Southwell. Study supervision: Berger.

\section{Correspondence}

Derek G. Southwell, Department of Neurological Surgery, University of California, San Francisco, 513 Parnassus Ave., M787, San Francisco, CA 94143. email: dereksouthwell@gmail.com. 